\title{
Conhecimento de profissionais de saúde do SUS acerca da paracoccidioidomicose no município de Valença-RJ
}

\author{
The knowledge of SUS health professionals about \\ paracoccidioidomycosis in the municipality of Valença-RJ
}

\author{
1 Thaís Helena Cassarotti thaiscassarotti@hotmail.com \\ 1 Thaynara Alves de Souza Maciel \\ 2 Luiz Henrique Conde Sangenis
}

1 Alunas de Iniciação Científica do Curso de Medicina de Valença, disciplina de Doença Infecciosas e Parasitárias. Faculdade de Medicina, Centro Universitário de Valença, UNIFAA.

2 Mestre e Doutor em Doenças Infecciosas e Parasitárias. Faculdade de Medicina, Centro Universitário de Valença, UNIFAA, disciplina de Doenças Infecciosas e Parasitárias

\section{Resumo}

A paracoccidioidomicose é a micose sistêmica mais importante da América Latina. Representa importante problema de saúde pública por ter alto potencial incapacitante, principalmente para determinadas parcelas da população, como os trabalhadores rurais, que apresentam grandes deficiências de acesso aos serviços de saúde. 0 presente estudo objetivou investigar o conhecimento dos profissionais de saúde que atuam no SUS do município de Valença-RJ a respeito da paracoccidioidomicose. Foi utilizado um questionário semiaberto com 10 perguntas sobre a paracoccidioidomicose, abrangendo a etiologia, modo de transmissão, epidemiologia, sintomatologia, diagnóstico e tratamento. Foram entrevistados 72 profissionais de saúde: 52 (72,2\%) médicos, 12 (16,7\%) odontólogos e oito $(11,1 \%)$ enfermeiros. Cerca de $60 \%$ dos médicos responderam não estar aptos para diagnosticar e tratar a doença. Entre odontólogos e enfermeiros, foi constatado grande desconhecimento acerca da doença. Entre os odontólogos, $67 \%$ afirmaram já terem ouvido falar da paracoccidioidomicose, no entanto nenhum deles sabia como diagnosticar e tratar. Entre os enfermeiros, 75\% nunca tinham ouvido falar da doença. Considerando o baixo conhecimento dos profissionais de saúde a respeito da paracoccidioidomicose revelado por este estudo e que o Brasil apresenta altas taxas de endemicidade, torna-se necessário investir no ensino e na capacitação de todos os profissionais de saúde que atuam no SUS para melhora da abordagem e do manejo da doença.

\section{Palavras-chave:}

Paracoccidioidomicose. Doença endêmica. Profissional de saúde. Conhecimento. Sistema Único de Saúde.

\begin{abstract}
Paracoccidioidomycosis is the most important systemic mycosis in Latin America. It represents an important public health problem because it has a high disabling potential, especially for certain population portion such as farm workers, who have major deficiencies in access to health services. This study aimed to investigate the knowledge of health professionals (doctors, dentists and nurses) that work in public health in the municipality of Valença-RJ regarding paracoccidioidomycosis. A semi-open questionnaire with 10 questions about paracoccidiodomycosis was used, covering the etiology, mode of transmission, epidemiology, symptomatology, diagnosis and treatment. A total of 72 health professionals were interviewed 52 (72.2\%) doctors, $12(16.7 \%)$ dentists and eight (11.1\%) nurses. About $60 \%$ of doctors said they were not able to diagnose and treat the disease. Among dentists and nurses, there was a great lack of knowledge about the disease. Among dentists $67 \%$ said they had heard of paracoccidioidomycosis, however none of them knew how to diagnose and treat it. Among nurses, $75 \%$ had never heard of the disease. Considering the low knowledge among health professionals about paracoccidioidomycosis revealed by this study and that Brazil has high rates of endemicity, it is necessary to invest in the education and training of all health professionals working in Unified Health System to improve the approach and the management of the disease.
\end{abstract}

\section{Keywords:}

Paracoccidioidomycosis. Endemic disease. Healthcare professional. Knowledge. Unified Health System.

\section{Como você deve citar?}

CASSAROTTI, Thaís Helena; MACIEL, Thaynara Alves de Souza; SANGENIS, Luiz Henrique Conde. Conhecimento de profissionais de saúde do SUS acerca da paracoccidioidomicose no município de Valença-RJ. Cadernos UniFOA, Volta Redonda (RJ), v. 16, n. 47, p. 121 - 128, dez, 2021. 
A paracoccidioidomicose (PCM) é uma micose causada por fungos termodimórficos do gênero Paracoccidioides spp., sendo reconhecidas duas espécies: $P$. brasiliensis e $P$. lutzii. A doença é endêmica e a principal micose sistêmica da América Latina, se estendendo do México até a Argentina, onde afeta, principalmente, trabalhadores rurais e indivíduos com atividades ligadas ao solo (SHIKANAY-YASUDA et al., 2017).

O Brasil concentra cerca de $80 \%$ dos casos relatados na literatura médica (WANKE; LONDERO, 1994). No estado do Rio de Janeiro (RJ), a doença é muito comum, tanto em áreas metropolitanas quanto em áreas rurais (MACEDO et al., 2017; VALLE et al., 2017). A cidade de Valença está inserida na região do Médio Paraíba do Sul, reconhecida como uma área de alta de endemicidade de PCM, englobando as cidades de Barra Mansa e Volta Redonda (GONÇALVES et al., 1998).

A PCM representa um importante problema de saúde pública por seu alto potencial incapacitante, principalmente para determinados segmentos, como os trabalhadores rurais, que apresentam grandes deficiências de acesso às unidades de saúde que prestam atendimento à doença. (SHIKANAI-YASUDA et al., 2017).

A PCM requer um diagnóstico precoce, fácil e disponível nas unidades primárias de saúde, de forma a contribuir para o início do tratamento em tempo adequado, o que reduz o risco de mortes e sequelas. Sendo a PCM uma micose sistêmica, na investigação diagnóstica, é essencial observar o estado geral do paciente e avaliar os principais órgãos e sistemas mais frequentemente comprometidos, diferenciando-se as formas clínicas da doença aguda/subaguda e crônica. (AMBRÓsıO et al., 2014)

A forma aguda/subaguda é responsável por 5 a $25 \%$ dos casos da doença, predominando em crianças e adolescentes, com distribuição semelhante entre os sexos. Essa forma clínica tem como característica uma evolução mais rápida e pode-se destacar a presença de linfonodomegalias, hepatoesplenomegalias, manifestações digestivas, lesões cutâneas e envolvimento osteoarticular como principais órgãos afetados (SHIKANAI-YASUDA et al., 2017).

Já a forma crônica, corresponde a 74 a $96 \%$ dos casos e se manifesta principalmente em adultos entre os 30 e 60 anos, predominando no sexo masculino. Tem progressão lenta e silenciosa, podendo levar muitos meses até o diagnóstico. As manifestações pulmonares estão presentes em $90 \%$ dos pacientes. 0 pulmão pode ser o único órgão afetado em até $25 \%$ dos casos. É considerada unifocal, quando a micose é restrita a um órgão, e multifocal, a mais comum, quando acomete mais de um órgão, sendo pulmões, mucosas das vias aerodigestivas superiores e pele os sítios mais acometidos pela infecção. (SHIKANAI-YASUDA et al., 2017).

Em alguns casos, os pacientes podem apresentar tanto manifestações da forma aguda/subaguda quanto da forma crônica e, nesses casos, são classificados como PCM forma mista. (SHIKANAI-YASUDA et al., 2017).

Nesse cenário, além de considerarmos as diversas formas de manifestação da doença, é necessário o conhecimento dos principais diagnósticos diferenciais, devendo-se incluir a PCM na investigação de diversas doenças comuns com sinais e sintomas semelhantes. Dentre elas, podemos citar como exemplos: tuberculose, linfomas, leucemias, toxoplasmose, leishmanioses, mononucleose infecciosa, histoplasmose, pneumonite intersticial, sarcoidose, hanseníase, neoplasias e cisticercose (WANKE; AIDE, 2009; SHIKANAI-YASUDA et al., 2017), atentando-se, para tanto, com a correlação desses diagnósticos com as principais características de cada forma de apresentação clínica da PCM. 
Valença é uma cidade situada na região do Médio Paraíba no sul fluminense com, aproximadamente, 76 mil habitantes, com altitude de $560 \mathrm{~m}$ e clima tropical. Na época do Brasil colonial, a região foi extremamente desmatada para o plantio de café. Com o declínio da produção cafeeira no século XX, muitas fazendas passaram a se dedicar a pecuária leiteira (IBGE, 2020).

De acordo com dados coletados nos registros do Hospital Escola de Valença, nos últimos cinco anos, ocorreram cerca de 2 a 3 casos novos ao ano de PCM no município, o que representa uma média de 2,6 a 3,9 casos por 100 mil habitantes, números que configuram alta endemicidade (SHIKANAIYASUDA et al., 2017).

Embora comum, há ainda grande desconhecimento a respeito da PCM, o que impõe uma série de desafios para o Sistema Único de Saúde (SUS). 0 diagnóstico é simples e a doença tem cura, porém a mortalidade ainda é elevada, impactada pelo baixo conhecimento da população e deficiências dos serviços de saúde. A ausência de estrutura para atendimento, de testes diagnósticos e de profissionais treinados nos serviços de saúde são alguns dos principais entraves para o combate da doença (FIOCRUZ, 2013).

Diante disso, o presente estudo objetivou investigar o conhecimento dos profissionais de saúde (médicos, odontólogos e enfermeiros) atuantes na rede SUS do município de Valença acerca da epidemiologia, suspeição, diagnóstico e tratamento da PCM.

\section{METODOLOGIA}

Trata-se de estudo descritivo transversal em que foi utilizado um questionário semiaberto elaborado especificamente para esta pesquisa. Foram formuladas 10 perguntas que abordaram a forma de transmissão, população mais afetada, diagnóstico clínico e tratamento da PCM. A investigação foi direcionada para os profissionais de saúde de nível superior - médicos, odontólogos e enfermeiros que atuavam na rede pública do SUS do município de Valença. Os questionários foram coletados de forma aleatória, presencialmente, de segunda a sexta-feira, no período de abril a novembro de 2019, por dois investigadores. Foram visitadas 10 unidades de assistência primária (UBS e ESF) e duas unidades hospitalares do município (setores de emergência, clínica, cirurgia, ginecologia/obstetrícia e pediatria). Todos os profissionais presentes no horário de trabalho em cada setor foram convidados a participar.

Para tabulação e análise dos dados, foi utilizado o Software Epi Info 2010 versão 3.5.2. Foram feitas análises descritivas de frequências percentuais, médias e medianas.

Este estudo foi submetido ao Comitê de Ética em Pesquisa (CEP) da Faculdade de Medicina de Valença - RJ, sob o número de CAAE 08685719.2.0000.5246 e número de aprovação 3.279.615. Todos os voluntários que aceitaram participar assinaram o Termo de Consentimento Livre e Esclarecido e foi garantido $o$ anonimato em todas as etapas da pesquisa.

\section{RESULTADOS}

Foram entrevistados 72 profissionais de saúde: 52 (72,2\%) médicos, 12 (16,7\%) odontólogos e oito $(11,1 \%)$ enfermeiros. Em relação ao local de atuação, 24 (33,3\%) atuavam na assistência primária (UBS e ESF) e $48(66,7 \%)$ na assistência hospitalar. 0 tempo médio de formado foi de 9,9 $\pm 6,0$ anos.

Dentre as áreas de especialização dos médicos, 11 (21,2\%) tinham formação em cirurgia; oito (15,4\%), em clínica médica; sete (13,5\%), em ginecologia/obstetrícia; cinco (9,6\%), em saúde da família; 
dois (3,8\%), em pediatria; seis (11,5\%), em outras áreas; e 13 (25\%) não tinham nenhuma especialização, sendo a maior parte dos participantes recém-formados. Entre os odontólogos, um (8,3\%) tinha formação em saúde da família; um (8,3\%), em odontopediatria; oito (66,7\%), em outras áreas; e dois $(16,7 \%)$ não tinham especialização. Já entre os enfermeiros, um $(12,5 \%)$ tinha especialização em saúde da família e sete $(87,5 \%)$ não tinham especialização.

Dentre os médicos entrevistados, apenas um $(1,9 \%)$ nunca tinha ouvido falar da PCM. Um terço dos odontólogos desconhecia a micose e, entre os enfermeiros, apenas dois (25\%) já tinham ouvido falar da doença. A maior parte dos médicos entrevistados $(71,2 \%)$ considerou a PCM comum em Valença. No entanto, a maioria dos odontólogos e enfermeiros não souberam responder. Quanto a indicar as pessoas mais afetadas pela PCM, $41(78,8 \%)$ médicos responderam que seriam os trabalhadores rurais; nove $(17,3 \%)$ indicaram os portadores de HIV/Aids; um $(1,9 \%)$, os moradores de comunidades pobres e um $(1,9 \%)$ não soube responder.

Metade dos médicos apontou a via inalatória como a principal forma de infecção da micose. A maior parte dos médicos respondeu não estar apta para diagnosticar $(59,6 \%)$ e nem tratar a doença $(55,8 \%)$, sendo que $59,6 \%$ não souberam informar o medicamento indicado no tratamento da PCM. 0 conhecimento dos profissionais de saúde de Valença acerca da PCM pode ser visto na Tabela 1. 
Tabela 1 - Conhecimento dos profissionais de saúde do SUS de Valença-RJ acerca da Paracoccidioidomicose.

\begin{tabular}{|c|c|c|c|c|}
\hline \multirow[b]{2}{*}{ Perguntas } & \multirow[b]{2}{*}{ Respostas } & \multicolumn{3}{|c|}{ Profissional/cargo (\%) N = 72} \\
\hline & & Médico & Odontólogo & Enfermeiro \\
\hline & & $52(72,2)$ & $12(16,7)$ & $8(11,1)$ \\
\hline \multirow{2}{*}{$\begin{array}{l}\text { Já ouviu falar da } \\
\text { PCM }\end{array}$} & Sim & $51(98,1)$ & $8(66,7)$ & $2(25)$ \\
\hline & Não & $1(1,9)$ & $4(33,3)$ & $6(75)$ \\
\hline \multirow{3}{*}{$\begin{array}{l}\text { A PCM é comum em } \\
\text { Valença }\end{array}$} & Sim & $37(71,2)$ & $3(25)$ & 0 \\
\hline & Não & $8(15,4)$ & $2(16,7)$ & 0 \\
\hline & Não sabe & $7(13,5)$ & $7(58,3)$ & $8(100)$ \\
\hline \multirow{5}{*}{$\begin{array}{l}\text { Como ocorre a } \\
\text { infecção (via) }\end{array}$} & Gravetos na boca & $25(48,1)$ & $6(50)$ & $1(12,5)$ \\
\hline & Inalatória & $26(50)$ & 0 & 0 \\
\hline & Picada de insetos & 0 & $1(8,3)$ & $1(12,5)$ \\
\hline & $\begin{array}{l}\text { Contato com } \\
\text { pessoas }\end{array}$ & $1(1,9)$ & $3(25)$ & 0 \\
\hline & Não sabe & 0 & $2(16,7)$ & $6(75)$ \\
\hline \multirow{2}{*}{$\begin{array}{l}\text { Sabe como } \\
\text { diagnosticar }\end{array}$} & Sim & $21(40,4)$ & 0 & 0 \\
\hline & Não & $31(59,6)$ & $12(100)$ & $8(100)$ \\
\hline \multirow{4}{*}{$\begin{array}{l}\text { Descrição dos } \\
\text { sintomas }\end{array}$} & Correta & $18(34,6)$ & 0 & $1(12,5)$ \\
\hline & Parcial & $24(46,2)$ & $1(8,3)$ & 0 \\
\hline & Errada & $1(1,9)$ & 0 & 0 \\
\hline & Não sabe & $9(17,3)$ & $11(91,7)$ & $7(87,5)$ \\
\hline \multirow{2}{*}{ Sabe como tratar } & Sim & $23(44,2)$ & 0 & $1(12,5)$ \\
\hline & Não & $29(55,8)$ & $12(100)$ & $7(87,5)$ \\
\hline \multirow{5}{*}{ Qual medicamento } & Itraconazol & $9(17,3)$ & 0 & $1(12,5)$ \\
\hline & Anfotericina B & $9(17,3)$ & 0 & 0 \\
\hline & Smx-tmp & 0 & 0 & 0 \\
\hline & Outros & $4(7,7)$ & 0 & 0 \\
\hline & Não sabe & $31(59,6)$ & $12(100)$ & $7(87,5)$ \\
\hline \multirow{2}{*}{$\begin{array}{l}\text { Tempo de } \\
\text { tratamento }\end{array}$} & Correto & $1(1,9)$ & 0 & 0 \\
\hline & Errado & $51(98,1)$ & $12(100)$ & $8(100)$ \\
\hline
\end{tabular}

Fonte: Dos autores

\section{DISCUSSÃO}

A PCM não faz parte do grupo de doenças tropicais consideradas negligenciadas pela Organização Mundial da Saúde (OMS), como a dengue, a doença de Chagas, as leishmanioses, a esquistossomose, a hanseníase, as treponematoses, a filariose, o tracoma, dentre outras (WHO, 2010). As únicas doenças causadas por fungos incluídas nessa lista de doenças tropicais negligenciadas são o micetoma, incluída, em 2016, e a cromoblastomicose, incluída em 2017 (QUEIROZ-TELLES et al., 2017). No entanto, a PCM comunga de características semelhantes das demais doenças negligenciadas, como o fato de atingir populações rurais empobrecidas, ser uma doença com pouca visibilidade, pouco conhecida e ocupar lugar secundário nas agendas de discussão de saúde (WHO, 2010). Mesmo assim, recente trabalho de revisão lista a PCM como uma das principais doenças fúngicas endêmicas negligenciadas da América Latina (QUEIROZ-TELLES et al., 2017). 
Apesar de comum e do Brasil concentrar a maior parte dos casos relatados, a PCM sequer faz parte da lista de doenças e agravos de notificação compulsória nacional, o que dificulta a implantação de medidas de vigilância e controle (MILLINGTON et al., 2018).

No presente estudo, foi observado grande desconhecimento do mecanismo de contágio, tendo em vista que $48,1 \%$ dos médicos e $60 \%$ dos odontólogos responderam que a transmissão ocorre pelo hábito de levar gravetos a boca. Apenas metade dos médicos (50\%) indicou a via inalatória como a principal via de transmissão. Nenhum odontólogo respondeu corretamente como ocorre a aquisição da PCM.

Entre os médicos, apenas um (1,9\%) afirmou desconhecer a doença e grande parte $(71,2 \%)$ sabia que a PCM era comum em Valença. Contudo, a maior parte dos médicos que atua na rede pública de saúde do município respondeu não estar apta a diagnosticar $(59,6 \%)$ e tratar $(55,8 \%)$ a PCM. Somente um terço dos médicos soube descrever corretamente os sintomas da doença e nomear medicamentos antifúngicos para o tratamento, sendo que uma das opções de medicamento mais utilizada no tratamento (sulfametoxazol-trimetoprim) sequer foi lembrada. Além disso, apenas um médico (1,9\%) soube informar o tempo correto de tratamento.

Deficiências de diagnóstico e no reconhecimento da PCM são fatores que podem impactar na morbidade e na letalidade. 0 pulmão é o órgão mais afetado e, muitas vezes, a doença é confundida com a tuberculose. No entanto, em até $15 \%$ dos casos, as duas doenças podem estar associadas (COUTINHO et al., 2015).

Nos últimos anos, as doenças tropicais negligenciadas vêm perdendo espaço nos currículos universitários. Pesquisa desenvolvida pela Fundação Oswaldo Cruz em parceria com a organização Médicos Sem Fronteiras, em seis municípios pertencentes à área endêmica da doença de Chagas no Brasil, demonstrou que apenas $32 \%$ dos profissionais de saúde conheciam os procedimentos diagnósticos e somente $14 \%$ sabiam indicar o tratamento correto da doença (Agência Brasil, 2018).

Chamou a atenção o elevado desconhecimento da PCM entre os demais profissionais de saúde investigados, particularmente os enfermeiros, pois $75 \%$ deles responderam nunca ter ouvido falar da micose. Ressalta-se que o enfermeiro, muitas vezes, é o primeiro profissional de saúde a ter contato com o paciente na unidade de saúde, particularmente na assistência primária (PEREIRA; FERREIRA, 2014), o que pode dificultar a identificação da doença.

No caso dos odontólogos, o desconhecimento da doença ainda envolve questões mais complexas. Um terço dos cirurgiões-dentistas entrevistados nunca tinha ouvido falar da PCM e nenhum deles se sentia apto para diagnosticar e tratar a doença. Resultado semelhante foi encontrado em pesquisa realizada em Mato Grosso, onde 63,5\% dos cirurgiões-dentistas responderam não estarem aptos para diagnosticar a PCM (ALMEIDA, 2014).

A maior parte dos pacientes acometidos de PCM apresenta comprometimento das mucosas da orofaringe, sendo a queixa de odinofagia um dos principais sintomas da forma crônica do adulto, o que leva o indivíduo doente a procurar inicialmente a assistência odontológica (BARROS et al., 2018; IKUTA et al., 2015). Em outras situações, a PCM pode ser confundida com neoplasias da boca, sendo o paciente, muitas vezes, encaminhado para avaliação odontológica (GIRARDI et al., 2012), o que aumenta a responsabilidade desse profissional no diagnóstico e tratamento da doença. 
O trabalho demonstrou um grande desconhecimento a respeito da PCM entre os profissionais de saúde. É necessário aprimorar a abordagem do tema nos currículos da graduação. Apesar de parte dos médicos e odontólogos demonstrarem algum conhecimento sobre a PCM, a maior parte demonstrou grande desconhecimento sobre o diagnóstico clínico e tratamento da doença. Cursos de capacitação poderiam contribuir para melhor abordagem e manejo da PCM por esses profissionais.

O grande desconhecimento geral sobre o tema demonstrado pelos profissionais de enfermagem sugere que a PCM não está fazendo parte do currículo desse profissional, devendo-se incluir ou melhorar a abordagem da doença durante a formação.

Considerando que o Brasil apresenta grande parte de seu território com altas taxas de endemicidade de PCM, torna-se necessáro aumentar a visibilidade sobre essa importante micose para todos os profissionais de saúde que atuam no SUS.

\section{AGRADECIMENTOS}

Agradecemos ao Centro de Pesquisa e ao Programa de Iniciação Científica (PROINC) do Centro Universitário de Valença - UNIFAA, pelo apoio para realização deste trabalho.

\section{REFERÊNCIAS}

AGÊNCIA BRASIL. Profissionais de saúde sabem pouco sobre doença de Chagas, diz estudo. Brasília, 2018. Disponível em: https://agenciabrasil.ebc.com.br/geral/noticia/2018-04/profissionais-de-saudesabem-pouco-sobre-doenca-de-chagas-diz-estudo. Acesso em: 20 out. 2020.

AMBRÓSIO, A.V.A.; CAMELO, C. C. S.; BARBOSA, C. V.; TOMAZATTI, F. G.; BRAZÕES, F. A. S.; VELOSO, J. M. et al. Paracoccidioidomicose (doença de Lutz-Splendore-Almeida): Propedêutica complementar, diagnóstico diferencial, controle de cura. Revista Médica de Minas Gerais. v.24, n.1, p. 81-92, 2014. doi: $10.5935 / 2238-3182.20140021$

ALMEIDA, H. L. Paracoccidioidomicose: perfil epidemiológico, clínico e terapêutico dos pacientes diagnosticados nos serviços de referência do Estado de Mato Grosso (2006-2013). Dissertação (Mestrado em Ciências da Saúde, Doenças Infecciosas e Tropicais). Universidade Federal do Mato Grosso, Cuiabá, 2014.

BARROS, L.; ASSIS, E. M.; GOMES, H. E.; SOUZA, P. E. A.; HORTA, M. C. R. Paracoccidioidomicose na mucosa oral: relato de caso. Revista Portuguesa de Estomatologia, Medicina Oral e Cirurgia Maxilofacial. v. 59, n. 3, p. 174-179, 2018. http://doi.org/10.24873/j.rpemd.2018.11.236

COUTINHO, Z. F.; WANKE, B.; TRAVASSOS, C.; OLIVEIRA, R. M.; XAVIER, D. R.; COIMBRA, C. E. Jr. Hospital morbidity due to paracoccidioidomycosis in Brazil (1998-2006). Tropical Medicine and International Health. v. 20, n. 5, p. 673-680, 2015. doi: 10.1111/tmi.12472

FIOCRUZ, Fundação Oswaldo Cruz. Doença rural negligenciada afeta 30 mil brasileiros. Agência Fiocruz de Noticias, 2013. Disponível em: https://agencia.fiocruz.br/doen\%C3\%A7a-rural-negligenciada-afeta-30mil-brasileiros. Acesso em: 08 nov. 2019. 
GIRARDI, F. M. SCROFERNEKER, M. L.; GAVA, V.; PRUINELLI, R. Head and neck manifestations of paracoccidioidomycosis: an epidemiological study of 36 cases in Brazil. Mycopathologia. v. 173, n. 2-3. p. 139-144, 2012. doi: $10.1007 / \mathrm{s} 11046-011-9488-5$

GONÇALVES, A. J.; Londero, A. T.; Terra, G. M.; Rozenbaum, R.; Abreu, T. F.; Nogueira, S. A. Paracoccidioidomycosis in children in the state of Rio de Janeiro (Brazil). Geographic distribution and the study of a "reservarea". Revista do Instituto de Medicina Tropical de São Paulo. n. 40, v.1, p. 11-13, 1998. doi: 10.1590/s0036-46651998000100003

IBGE, Instituto Brasileiro de Geografia e Estatística. Valença (RJ)/ Cidades e Estados, 2020. Disponível em: https://www.ibge.gov.br/cidades-e-estados/rj/valenca.html. Acesso em: 12 set. 2020.

IKUTA, C. R. S.; TIEGHI-NETO, V.; IMADA, T. S. N.; LIMA, H. G.; LARA, V. S.; SANTOS, P. S. S. Paracoccidioidomicose crónica: caraterísticas intraorais em um relato de caso clínico. Revista Portuguesa de Estomatologia, Medicina Oral e Cirurgia Maxilofacial. v. 56, n. 4, p. 246-250, 2015. http://dx.doi.org/10.1016/j. rpemd.2015.08.003

MACEDO, P. M.; ALMEIDA-PAES, R.; FREITAS, D.F.; VARON, A. G.; PAIXÃO, A. G.; ROMÃO A. R. et al. Acute juvenile Paracoccidioidomycosis: A 9-year cohort study in the endemic area of Rio de Janeiro, Brazil. PLoS Neglected Tropical Diseases. v. 11, n. 3, p. e0005500, 2017. doi: 10.1371/journal.pntd.0005500.

MILLINGTON, M. A.; NISHIOKA, S. A.; MARTINS, S. T.; SANTOS, Z. M. G. D.; LIMA-JÚNIOR, F. E. F.; ALVES, R. V. Paracoccidioidomicose: abordagem histórica e perspectivas de implantação da vigilância e controle. Epidemiologia e Serviços de Saúde. v. 27 n. esp, p. e0500002, 2018. doi: 10.5123/S1679-49742018000500002

PEREIRA, R. T. A.; FERREIRA, V. A consulta de enfermagem na Estratégia Saúde da Família. Revista UNIARA. v. 17, n. 1, p. 99-111, 2014.

QUEIROZ-TELLES, F.; FAHAL, A. H.; FALCI, D. R.; CACERES, D. H.; CHILLER, T.; PASQUALOTTO, A. C. Neglected endemic mycoses. Lancet Infectious Diseases. v.17, n. 11, p. e367-e377, 2017. doi: 10.1016/ S1473-3099(17)30306-7

SHIKANAI-YASUDA, M. A.; MENDES, R. P.; COLOMBO, A. L.; QUEIROZ-TELLES, F.; KONO, A. S. G.; PANIAGO, A. M. M. et al. Brazilian guidelines for the clinical management of paracoccidioidomycosis. Revista da Sociedade Brasileira de Medicina Tropical. v. 50, n. 5, p. 715-740, 2017. doi: 10.1590/0037-8682-0230-2017

VALLE, A. C. F.; MARQUES DE MACEDO, P.; ALMEIDA-PAES, R.; ROMÃO, A. R.; LAZÉRA, M. D. S.; WANKE, B. Paracoccidioidomycosis after Highway Construction, Rio de Janeiro, Brazil. Emerging Infectious Diseases. v. 11, p. 917-1919, 2017. doi: 10.3201/eid2311.170934

WANKE, B.; AIDÊ, M. A. Chapter 6 - Paracoccidioidomycosis. Jornal Brasileiro de Pneumologia. v. 35, n. 12, p. 1245-1249, 2009.

WANKE, B.; LONDERO, A. T. Epidemiology and paracoccidioidomycosis infection. In: Franco MF, Lacaz CS, Restrepo-Moreno A, Del Negro G, editors. Paracoccidioidomycosis. Boca Raton: CRC Press, 1994. p. $109-120$.

WHO, World Health Oganization. First WHO report on neglected tropical diseases: working to overcome the global impact of neglected tropical diseases. Geneva, World Health Organization, 2010. 